\title{
Human-Controlled Production
}

\author{
Matthias Neubauer, Florian Krenn, loan-Alexandru Schärfl, \\ Christian Stary and Dennis Majoe
}

\begin{abstract}
In factories of the future, the worker and his or her well-being is regarded a crucial part of manufacturing situations. Human factors are recognized as vital to achieve sustainable organizational success. Advances in the area of wearable sensors proclaim that sensing human properties within manufacturing settings is technically feasible. Thereby, sensing human properties, such as the level of comfort or stress, may be used to adapt system behaviour in manufacturing situations. This chapter revisits related work from adaptive systems design addressing triggers for adaptations and impacted dimensions. The related work can be considered as design space for developers of S-BPM-based adaptive processes. In line with the related work, a laboratory setting at the Johannes Kepler University Linz has been designed and utilized for testing sensor-based process behaviour and control. Essential findings are described with respect to system architectures and S-BPM process design. The chapter concludes with relating modelling adaptive to human-aware S-BPM processes on a concept layer, and future work.
\end{abstract}

M. Neubauer $(\bowtie) \cdot$ F. Krenn $\cdot$ I.-A. Schärfl · C. Stary

Department of Business Information Systems - Communications Engineering,

Johannes Kepler University Linz, Linz, Austria

e-mail: matthias.neubauer@jku.at

D. Majoe

MA Systems and Control Limited, Southampton, UK 


\subsection{Related Work}

In factories of the future, humans represent a crucial part for increasing flexibility, agility and competitiveness. Today, the achievement of manufacturing objectives relies inter alia on the seamless interaction among humans and machines. The research field of adaptive systems investigates how joint human-machine systems may "change their behaviour to meet the changing needs of their users" (Feigh et al. 2012). A prerequisite for the selection of adequate changes is the assessment of the user's current situation (cf. Fig. 6.1). The advent of IT and sensor technology has enabled the assessment of human properties in order to align the interactions among humans and machines.

However, yet the design and implementation of adaptive systems remains a challenging task. In this chapter, the applicability of S-BPM for the design and implementation of human-aware adaptive production systems is investigated.

The design space and respective guidelines in the field of adaptive systems are revisited subsequently. Figure 6.1 illustrates the elements of a generic adaptive system according to Feigh et al. (2012). It comprises a "perceive, select, and act" cycle. A "Context Assessment" component is responsible for perceiving certain changes in different types of states, e.g. human state, system state and task/mission state. Based on the perceived state, the "Adaptions Manager" is responsible for selecting appropriate system adaptations, e.g. rescheduling of tasks or new

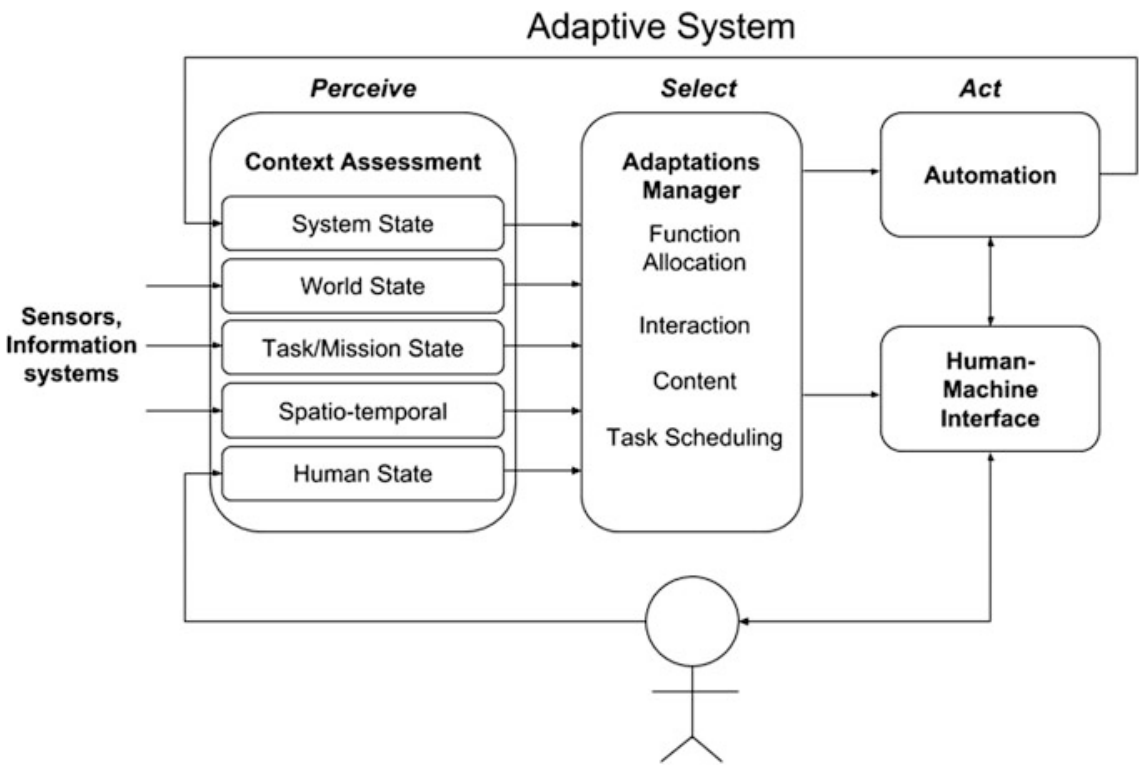

Fig. 6.1 Generic adaptive joint human-machine system (adapted from Feigh et al. 2012, (C) Human Factors and Ergonomics Society) 
allocation of functions. These changes may affect the current system actions in terms of modified automated system behaviour or changes of the human-machine interface.

Feigh et al. (2012) present a twofold framework for adaptive systems containing: (1) triggers for adaptation and (2) types of adaptation. Adaptations may be triggered by operators, the system state or mode, a certain environmental state or event, a task state or mission event or a spatio-temporal event (cf. Fig. 6.2).

Operator-based triggers can be directly initiated by operators; in other words, humans control system adaptations through their input. Furthermore, states of operators can be measured by system components and trigger changes. For instance, a tiredness sensor in a car observes the driver and triggers an audio warning signal when it detects the risk of micro-sleep.

System-based triggers can originate from a change of the system state or mode. Feigh et al. (2012) refer to system state as "description of the current configuration of the automation", e.g. a smart home may trigger different behaviours depending on the state "Cooling | Heating" with respect to the sun-blinds. In case "Cooling" is activated, the temperature in a room is above a certain threshold, and the sun is shining, the sun-blinds will go down. A system mode represents a group "of several system configurations under one label where typically each mode corresponds to a set of unique system behaviours" (Feigh et al. 2012 cited in Johnson 1990).

Environmental-based triggers refer to triggers that occur due to environmental changes being external to the operator or system components. Typical examples for such changes are changing light level, temperature, humidity or wind.

Task- and Mission-based triggers occur when a certain task, e.g. "drill part" or a mission "produce high precision lot-size 1 part" is accomplished. The distinction between task and mission is stated by Feigh et al. (2012) as "A mission is typically organized into phases or subgoals, each of which is subject to constraints such as the time to complete and pre-and postconditions. Adaptation management based on task state uses the initialization, completion or partial completion of tasks (regardless of their impact on mission goals or objectives) to drive changes in automation".

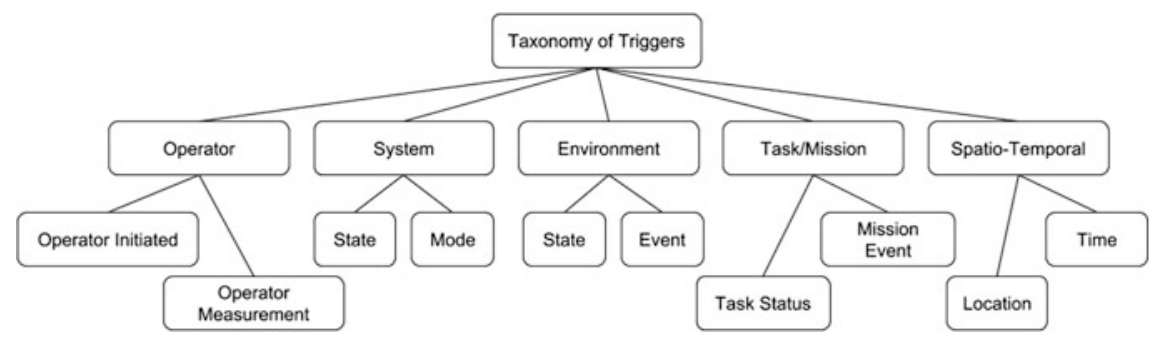

Fig. 6.2 Taxonomy of Triggers for adaptive systems (adapted from Feigh et al. 2012, (C) Human Factors and Ergonomics Society) 
Spatio-temporal triggers represent system changes that are triggered based on certain times or location, e.g. every day at 7 o'clock the sun-blinds go up, after 10 min of full speed a machine stops to ensure long lifetime and when a qualified employee is near a machine that need to be maintained, he/she will be notified.

The triggers given above may result in different types of adaptations as depicted in Fig. 6.3. The adaptation types sketch the system elements that can be adapted when a certain trigger occurs. For example, stress measurement of operators could trigger task reallocation.

In their taxonomy, Feigh et al. (2012) identify four fundamental elements that may be affected: (i) Function Allocation, (ii) Task Scheduling, (iii) Interaction and (iv) Content.

Function Allocation refers to mechanisms that determine who (human or machine) is responsible to perform a certain function or task. In the literature, static and dynamic function allocations are differentiated. In case of static function allocation, the assignments are defined at design time, whereas in the case of dynamic function allocation the assignment may change during runtime. Such assignments need to consider responsibility and authority of system agents. From an operator's point of view, the modification of function allocation gets evident with respect to task sharing and task offloading. According to Feigh et al. (2012), task sharing refers to the division of work between operators and automated systems, and task offloading aims to shift tasks from operators to automated system elements. However, sharing and offloading may also occur among different operators and not solely between operators and machines.

Task Scheduling considers the timing, duration and prioritization of tasks to be executed by a system. Timing refers to the point in time when a certain task is instantiated. The priority of a task can be differentiated by urgency and importance. Urgency depends, e.g. on the time available to respond or the certainty of a given information. Task importance refers to the potential impact of task failure, e.g. arising safety issues. Aside from the timing and the prioritization, tasks take a certain amount of time. The duration of a task may vary depending on tools used, skills of workers or other contextual factors (e.g. temperature at shop floor) (Feigh et al. 2012).

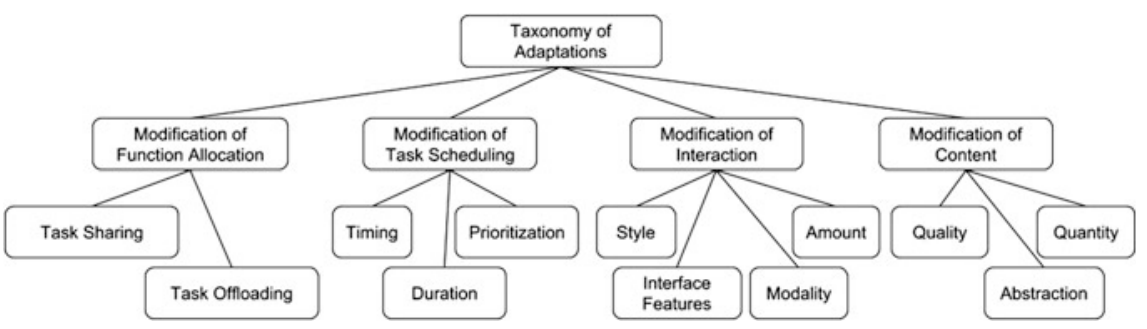

Fig. 6.3 Taxonomy of adaptations for adaptive systems (adapted from Feigh et al. 2012, (C) Human Factors and Ergonomics Society) 
Modification of Interaction focuses on the adaptation of human-machine interaction. According to the taxonomy, such modifications can affect interface features, interaction styles, the frequency of interaction and the modality of communication. The modification of interface features affects the layout or the navigation means provided to humans. When considering the frequency of interaction between humans and machines, one needs to define how much interaction at which point in time is required. Modality of communication refers to the adequate communication channel between humans and machines, e.g. a machine could simply display information or provide the information via text-to-speech means in an auditory way. Interaction styles define "rules of engagement", e.g. pro-active or re-active communication patterns between humans and machines.

Modification of Content considers the modification of content exchanged between machines and humans. Thereby, content may vary with respect to quantity, the level of abstraction or quality. The quantity of content addresses the amount of information displayed. For example, on mobile devices typically less information is displayed compared to larger screens of desktop PCs. Content may also be modified regarding the degree of abstraction. Information may be aggregated to support human operators and reduce processing times. Vice versa, it may be provided in a more detailed form if necessary. Furthermore, Feigh et al. (2012) consider the modification of the quality of content relevant for system adaptation, e.g. videos or pictures may be shipped with different resolutions depending on device and network connection.

The framework of Feigh et al. (2012) aims to provide a guideline for designers, evaluators and researchers when building, evaluating or researching adaptive system behaviour. In addition to Feigh et al. (2012), Steinhauser et al. (2009) propose design guidelines for adaptive automation:

1. Adaptive function allocation should be used intermittently

2. Energetic human qualities should be considered in design

3. Emotional requirements of the human operator need to be considered

4. The system should be calibrated to the individual operating it

5. Task transformation should be used to simplify tasks for operators

6. The environmental context of the system should be used to determine allocation

7. Tasks should be partitioned when both the human and the system can contribute effectively

8. Adaptation should be controlled by the system, but be open to human intervention when the system fails to recognize new conditions or demands

Beyond guidelines, the well-being of humans plays a crucial in design. Findings from occupational psychology reveal "the number of employees experiencing psychological problems related to occupational stress has increased rapidly in Western countries" (Van der Klink et al. 2001, p. 270). Basically, stress can be measured by applying (i) psychological questionnaires (ex-post to stressful situations) or (ii) by measuring physiological measures like heart rate variability or 
blood pressure (cf. Taelman et al. 2009; Lawler 1980; Kelsey 1991). The latter, in combination with wearable sensors, enables live sensing of human stress levels in certain work situations. Such live data provide a basis for analyzing human conditions in work situations, adapting system behaviours at runtime and informing workplace (re-)design activities. For example, tasks could be reallocated based on the current workload and stress level of workers, process steps in which a high stress level occurs could be investigated and redesigned or individuals could monitor and self-assess their own stress level in order to increase their stress-awareness.

A detailed investigation of the efficacy of psychophysiological measures for implementing adaptive technology is presented, e.g. by Screbo et al. (2001). They reviewed physiological measures, such as Eye Blinks, Respiration, Cardiovascular Activity, and beyond that, cortical measures like EEG Bandwidths: Arousal, Attention and Workload; EEG and Biofeedback; EEG Biofeedback and Task Performance; Event Related Potentials; Cerebral Metabolism and Blood Flow.

In the subsequent sections, human-aware S-BPM designs are explored taking into account physiological measures and guidelines for adaptive systems design.

\subsection{Stress-Aware Lego Assembly}

Today's factories employ people to perform the semi-automatic assembly of a variety of mechanical and electro-mechanical products. Increasingly, the assembly lines are run lean and just in time. When products are built to incoming orders, factories typically perform activities such as: production, packaging and shipping. Production of a single product may be achieved by a single assembly person or a line of them. Once a unit is complete, it needs to be tested, quality checked and then packed. The wrapped parcel then needs to undergo relevant customs and shipping formalities.

The workflow has the conveyor belt and the workers at its centre. Incoming orders need to be shipped as soon as possible. However, enabling workers to perform at an optimum rate (high productivity, high quality, low errors and high job satisfaction) needs to consider a number of critical factors:

- Is there sufficient challenge in the job?

- Is there insufficient challenge leading to long idle times and too much boredom?

- Is the quality of assembly meeting the required standards?

- Is the accuracy (e.g. shipping docs) $100 \%$ ?

- Is the workflow under the control of the worker, or is it reverse?

- Stress may arise due to a number of factors; is the level of stress at acceptable limits?

- Is the job challenge/boredom/stress level of one person the same as that of another person? 
The work rate on a production line and the error rate (or qualities achieved) are the most important factors for the factory management. Working the line at high rate for long periods will inevitably result in costly errors and worker dissatisfaction.

There are two control points in the workflow: raising a new order and the rate of flow of the conveyor. Using statistical averages, for a given order input rate, theoretically, the conveyor belt should run at a given rate for a given level of quality. However, this statistical rule does not take into account the individuality of the workers who may swap roles on the shop floor and who may achieve higher productivity, as they balance their challenge and boredom levels across several roles at several different times.

When new parts for assembly are introduced, workers may need much more time than statistically allowed for, so that they can become familiar with a particular part. The continuous roll of the conveyor belt can also cause stress for workers simply because (apart from emergency cases) they do not control the conveyor belt per se. Handing over some level of control to the worker slowing down or stopping the belt could empower the worker.

Stress on the production line has been investigated in different research. A study by Lundberga et al. (1989) shows that perceived stress at an assembly line is consistently reflected in cardiovascular and neuroendocrine functions of the workers. Work induced a significant elevation in almost all psychological and physiological measurements. Levels were consistently lower in workers reporting a 'good' workday compared to those reporting a 'normal' or a 'bad' day. Correlations between self-reports and physiological values showed that catecholamine and cortisol responses, respectively, tend to be associated selectively with different psychological conditions, catecholamine values being associated with feelings of time pressure and pressure by demands, cortisol values with irritation, tenseness and tiredness.

Catecholamines are released into the blood when a person is under physical or emotional stress. Catecholamines increase heart rate, blood pressure, breathing rate, muscle strength and mental alertness. They also lower the amount of blood going to the skin and intestines and increase blood going to the major organs, such as the brain, heart and kidneys.

It has been shown by Jacobs et al. (1994) that skin conductance level should be useful in studies assessing the impact of mental stress on cardiovascular function. First, measures of electrodermal activity such as skin conductance level have been found to be highly suitable for monitoring autonomic nervous system activity because such an activity is determined by the sympathetic branch of the autonomic nervous system, which is predominant in stress. Second, as with indexes of cardiac performance such as blood pressure and heart rate, electrodermal response has been found to reliably increase during laboratory mental stress (Boblin 1976; Kelsey 1991; Lawler 1980; O’Gorman and Homeman 1979) and under other threatening, novel or challenging conditions. 

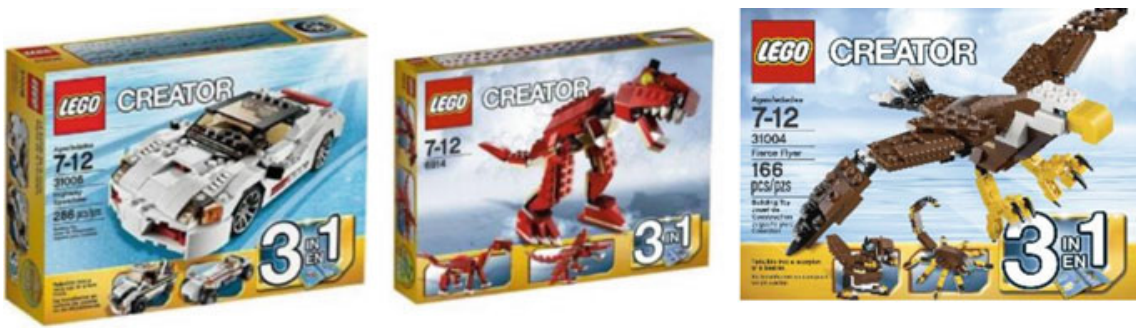

Fig. 6.4 LEGO creator building kits

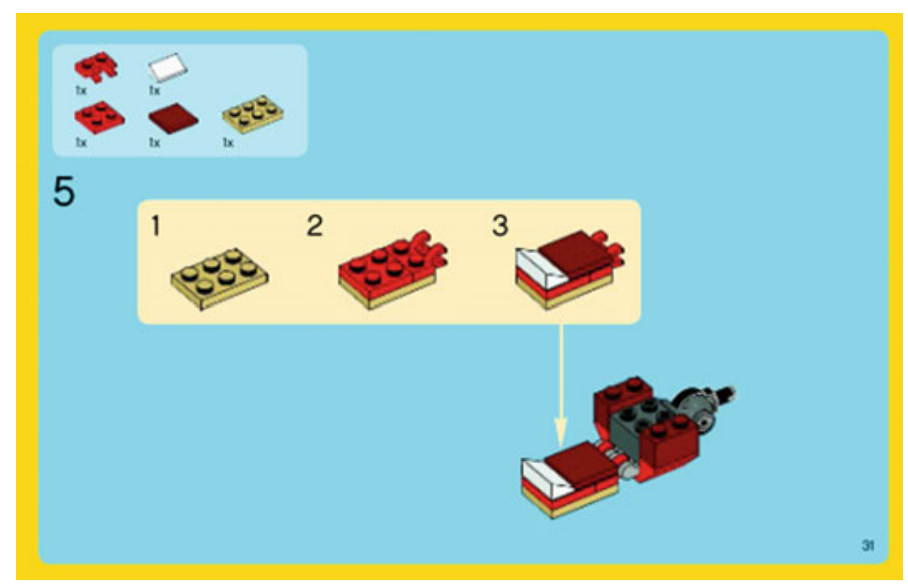

Fig. 6.5 LEGO creator building instructions. Source LEGO Group. http://lego.brickinstructions. com/lego_instructions/set/6914/TRex

Jouven et al. (2009) found when working with 5713 subjects that the mean heart rate increased during mild mental stress was $8.9 \pm 10.8$ b.p.m. (beats per minute) and the cut-off values of the tertiles of heart rate increase during mild mental stress were, 4 b.p.m., between 4 and 12 b.p.m., and above 12 b.p.m. Therefore, in order to assess the stress on a worker over periods of time, we propose that sensors are used to measure heart rate, blood pressure and skin conductance.

By combining data from these sensors and calibrating them for different workers, the work stress may be estimated and indicated. Subsequently, a laboratory study is presented in which test persons assemble different types of LEGO creator models supported by dedicated building instructions (cf. Figs. 6.4 and 6.5). Thereby, their heart rate is measured and the stress level is indicated via a PLC control LED. 


\subsubsection{Assembly Workplace Setup}

The LEGO assembly workplace is sketched in Fig. 6.6. It comprises the following elements:

- Multiple Boxes for assembly orders containing

- LEGO bricks

- Order sheet with QR-Code

- A webcam to identify an order and the related assembly task

- A monitor to display building instructions

- Three LED lights (green, yellow, red) to indicate stress level (low, medium, high)

- The BioRing sensor to measure heart rate, skin conductance, accelerometer data of test persons

- An iPod touch receiving the BioRing data and forwarding them to the S-BPM processing system

The test sessions were structured as follows:

- Introduction of laboratory test and assembly workplace to test person

- Equipping test person with BioRing sensor and initializing of iPod Touch app and S-BPM processing system

- 5 min baseline measurement of test persons in calm state

- 20 min LEGO assembly

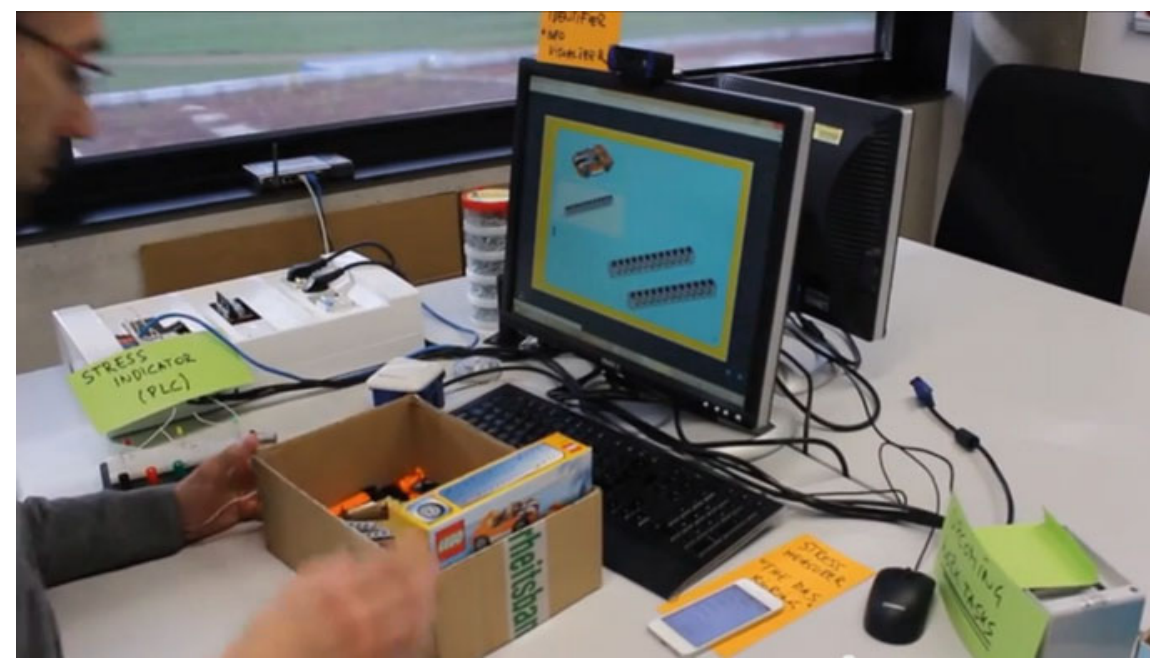

Fig. 6.6 Laboratory LEGO assembly workplace 
When building certain assembly order, the building instructions were displayed at the screen. In advance to the tests, an experienced LEGO builder assembled the test order and his timing for certain assembly instructions was measured. This reference timing was used as target time for test subjects who were not experienced in building the given types of LEGO models. The target timing and different model variants were intended to challenge test persons and simulate stressful situations.

\subsubsection{S-BPM Implementation}

Figure 6.7 depicts the implemented system architecture for the LEGO assembly test case. The architecture intertwines the BioRing Sensor, dedicated S-BPM processes implemented in Metasonic Suite and Programmable Logic Controller (PLC) for indicating stress levels. In the given architecture, the Metasonic workflow execution system MFlow comprises the logic for data interpretation and context-sensitive behaviour execution. Thereby, sensor data related to a workplace can be provided by both individual human sensors and the shop floor related low-level controls (cf. PLC in Fig. 6.7). However, in the given scenario the PLC mainly acts as indicator and not as sensor.

The interaction among the system components depicted in Fig. 6.7 can be described as follows. Sensor data are associated with a unique identifier linked to a specific worker. This information is required, in order interpret measured live data with respect to individual baseline and furthermore indicate levels at the workplace where the actual workers remain. A sensor has neither a direct interface to the S-BPM process execution environment nor the low-level controls. The measured data is instead sent to a mobile device using Bluetooth 4.0 and a low-level protocol. This allows for exploiting the connectivity of mobile devices (e.g. WiFi).

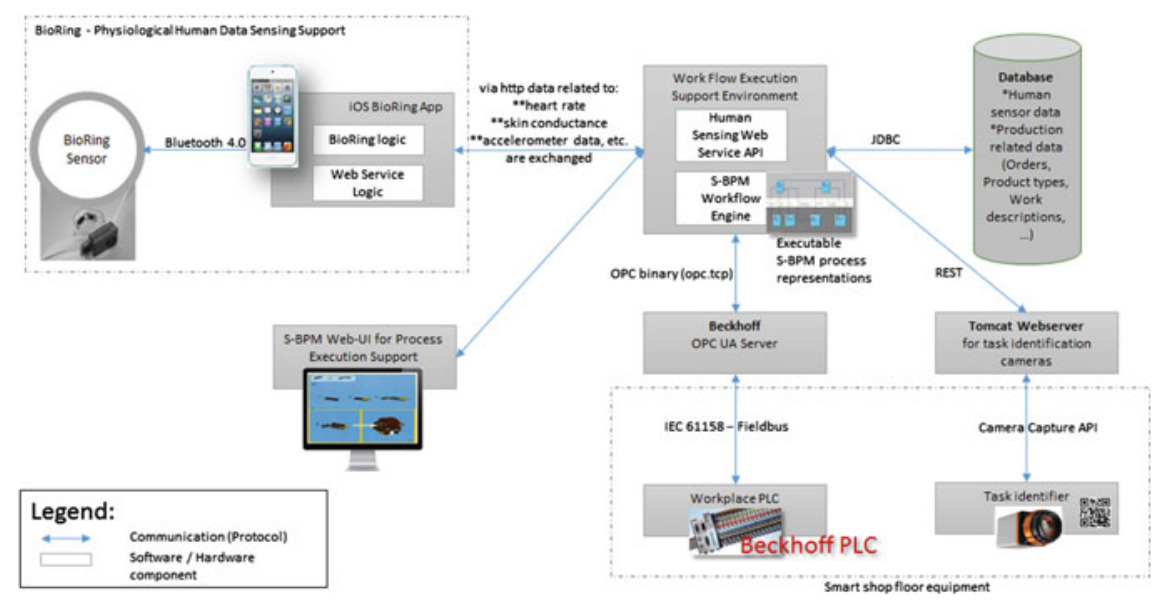

Fig. 6.7 System components for stress measurement and indication when assembling LEGO 
Furthermore, a pre-processing (e.g. aggregation, buffering) of the measured data can be done on the mobile device. Additionally, the mobile device may provide worker-related data, e.g. name, location or motion, relevant for context-sensitive process execution.

To forward the pre-processed data to the workflow execution system, a mobile application is used. This mobile application may interface the workflow system via an API (e.g. Web Service API). In the given application, the communication is mediated via a "Human Sensing Web Service API", which encapsulates the data low-level data exchange protocol used by the BioRing and provides means for the S-BPM workflow system to access individual data on a higher degree of abstraction. Based on the sensor data, the workflow system may adapt its process behaviour or trigger changes at PLCs. In the implemented architecture, the communication between Metasonic Flow and the Beckhoff PLC is realized using OPC UA (OLE for Process Control Unified Architecture, cf. https://opcfoundation. org/) to change different stress indication modes.

The implemented S-BPM process design for the LEGO Assembly is described in the following. Thereby, the process logic has been divided into two processes: (i) the LEGO assembly process and the (ii) stress measurement and indication process. The LEGO Assembling subject (cf. Fig. 6.8) represents the start subject and triggers the start and end of an assembly session. When triggering such a session, the Task Identifier subject and the Stress Measurement subject are notified via dedicated messages.

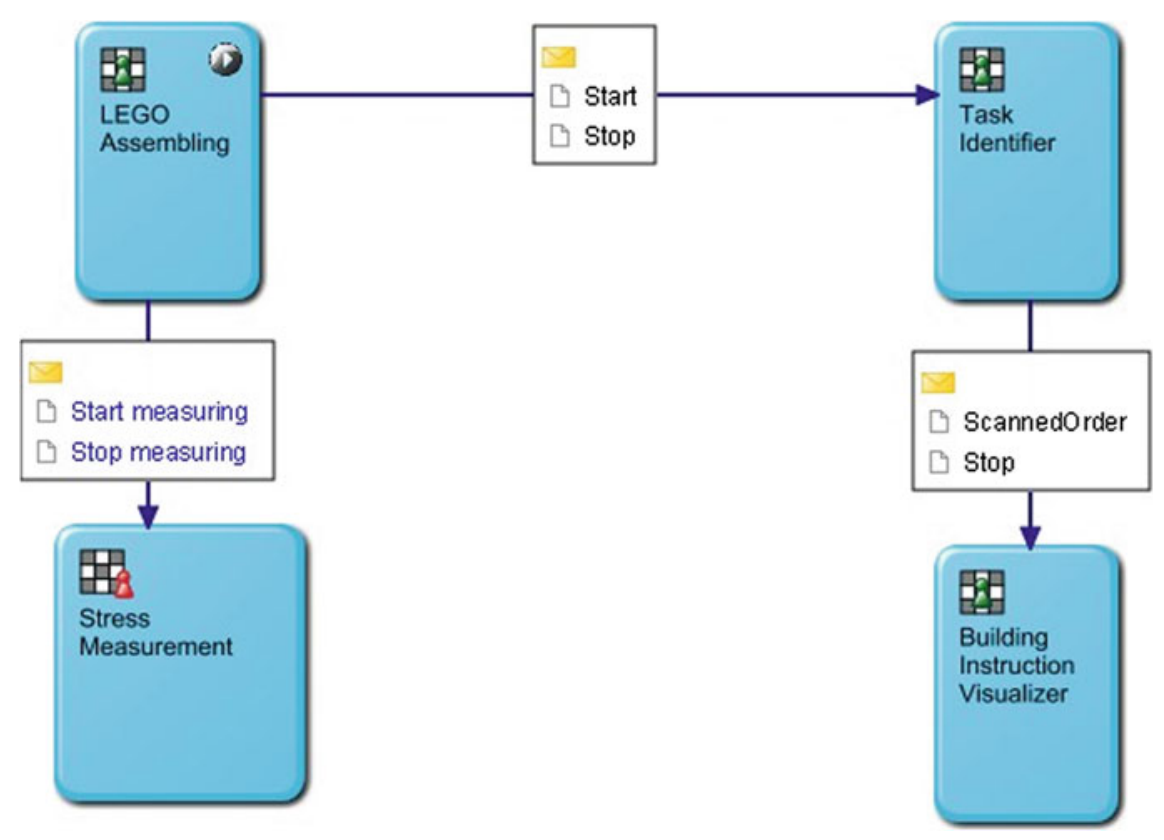

Fig. 6.8 SID—Lego Assembling process 
The Task Identifier subject exhibits the behaviour for scanning a QR-code and triggering the Building Instruction Visualizer. Figure 6.9 depicts the subject behaviour of the Task Identifier. Upon receiving the start message from LEGO Assembly, Task Identifier proceeds with scanning QR-codes. Within the function state 'Scan code', a refinement (custom java code) is executed that accesses the camera via a dedicated camera API and reads a QR-code. The QR-code corresponds to orderIds, and thus supports to identify which building instructions shall be displayed and in which state of production a certain assembly order currently remains. In case a QR-Code is successfully read, the scanned orderId is sent to the 'Building Instruction Visualizer'. At any point in time a 'stop' message from LEGO Assembling may be received, which terminates the task identification behaviour. This event is realized via the message event handler 'abort' in Fig. 6.9

The 'Building Instruction Visualizer' subject acts upon the receipt of 'ScannedOrder' messages from the Task Identifier. The message comprises the orderId that allows querying the relevant data from the database in the 'Retrieve order details' function. Based on the queried data, a custom refinement retrieves relevant building instructions for the current order and displays them in a temporal sequence on the screen. When completing the given assembly step, the order is updated and the next scanned order will be displayed (Fig. 6.10).

In parallel to the LEGO Assembling process support, a Stress Measurement and Indication process will be executed for each worker. This process is triggered or terminated by the LEGO Assembling subject via dedicated start and stop messages. The Stress Measurement subject itself will further notify the Stress Indicator subject about start or stop messages and changing stress levels of the test person (Fig. 6.11).
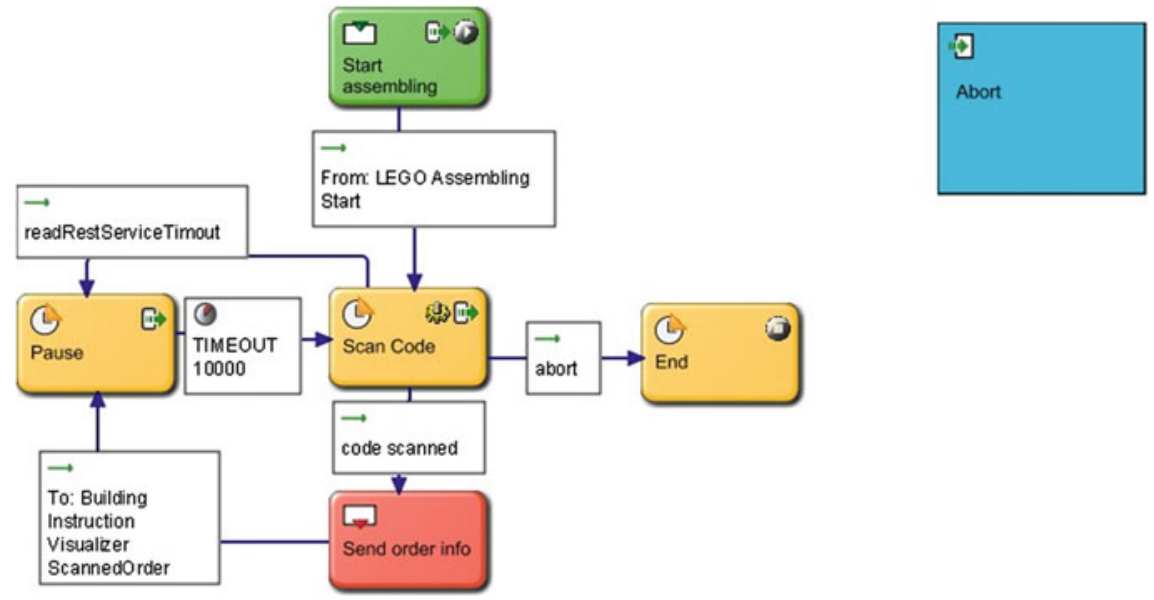

Fig. 6.9 SBD-Task Identifier subject 


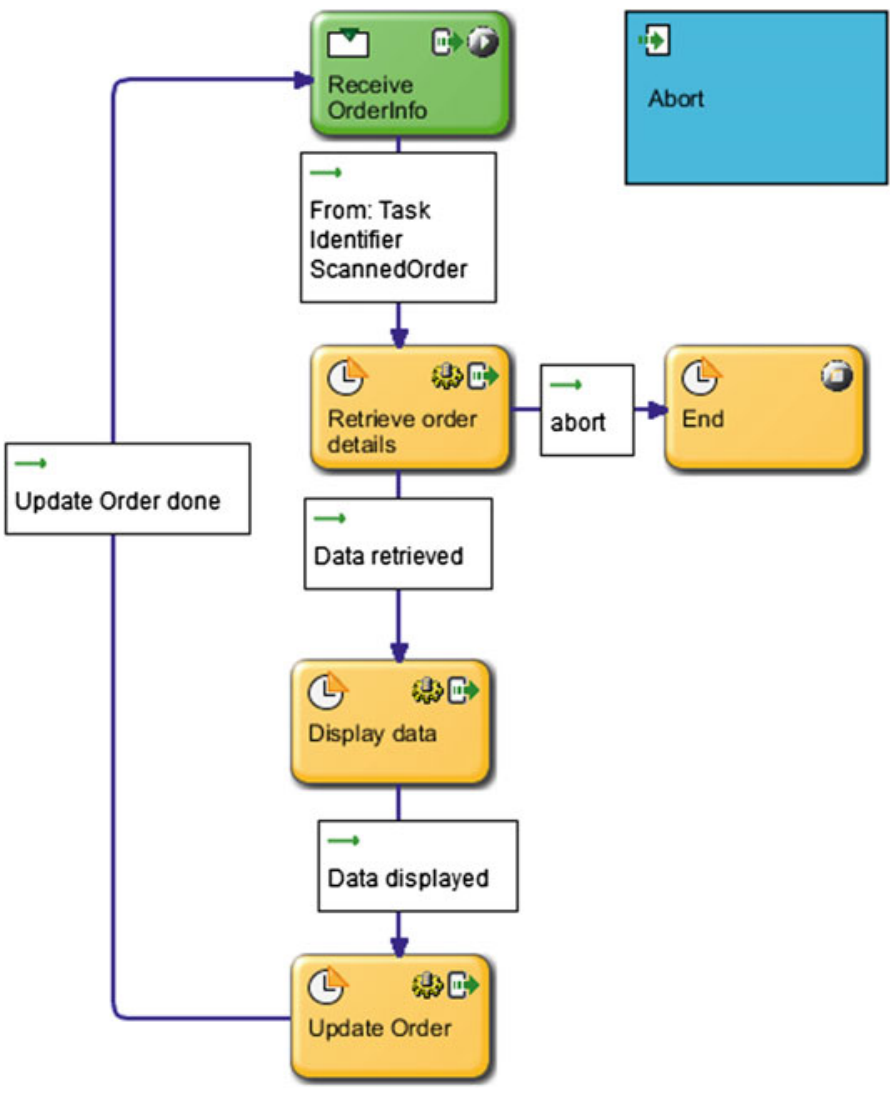

Fig. 6.10 SBD—Task visualizer

The internal behaviour of the Stress Measurement subject is presented in Fig. 6.12. The S-BPM process and the sensor data are intertwined via a database storing human physiological data. Thus, equipping the test person with the sensor and running the App on the iPod touch for communicating the measurements to the human physiological database is a prerequisite for the process execution. Within the process, the 'Stress measurement' subject determines within the initial $5 \mathrm{~min}$ the individual baseline of the test person. Subsequently, the stress level of a test person is evaluated on a regular basis (in Fig. 6.12, for example, each and every $8 \mathrm{~min}$ ) and either 'low', 'medium' or 'high' is sent to the 'Stress Indicator' subject. Again, this process may be terminated at any point in time via receiving a 'Stop measuring' message within the Abort message event handler.

The 'Stress Indication' subject defines the behaviour when changes in stress levels of a test person arise. Thereby, the subject interfaces the PLC via a configured OPC UA server and dedicated OPC UA refinements for different stress levels (cf. Indicate low, medium and high stress function states in Fig. 6.13). An extension 


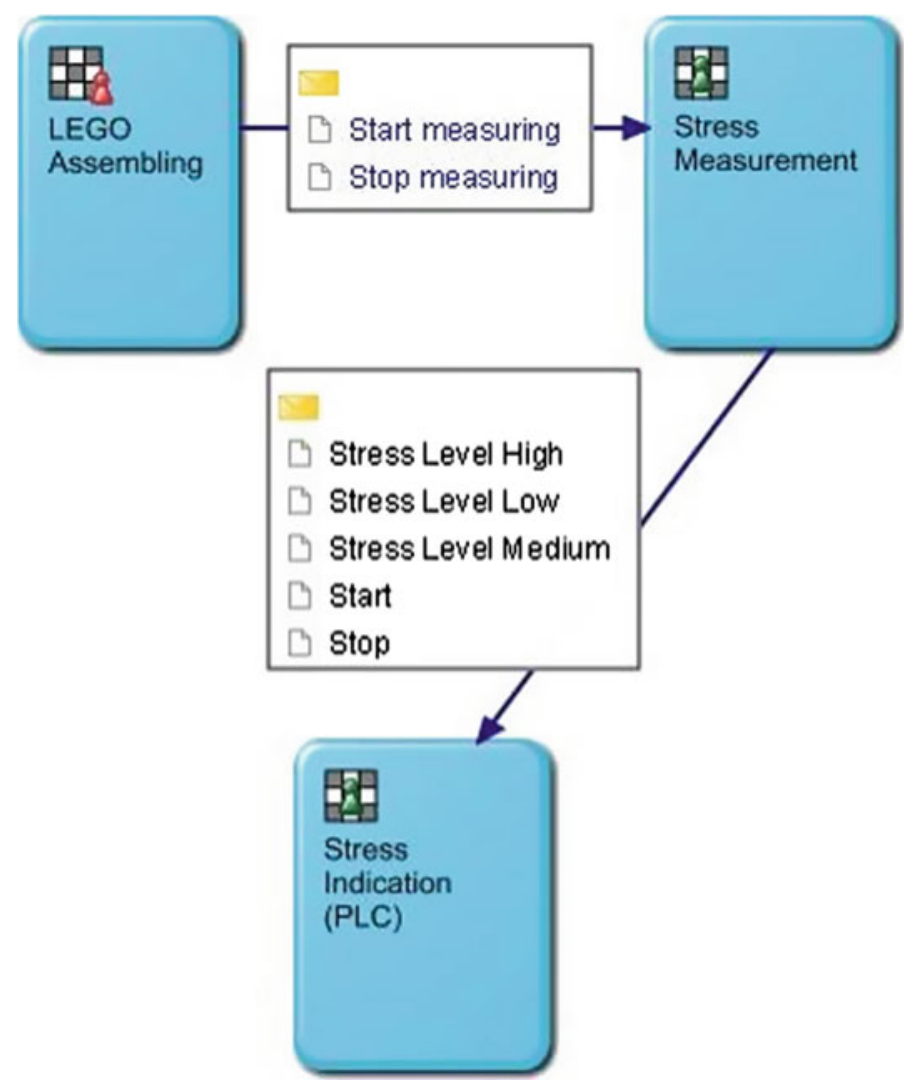

Fig. 6.11 SID - Stress Measurement and Indication process

to Metasonic Suite provides OPC UA refinement templates enabling to get/set values on a PLC based on business object values in the Metasonic Suite. For example, in Metasonic Suite, a business object 'StressIndication' may exist that includes three Boolean values (i) green ON, (ii) yellow ON and (iii) red ON. In case the stress level is low, the values of the business object should be green $\mathrm{ON}=$ true, yellow $\mathrm{ON}=$ false, red $\mathrm{ON}=$ false. These values may then be transferred to the target PLC using the OPC UA template (cf. Fig. 6.14 OPC UA template).

\subsubsection{Findings}

\subsubsection{Measuring Human Physiological Data in Work Situations}

Within the SO-PC-Pro project, the team aimed to investigate the measurement of human physiological data with the BioRing sensor, a research prototype developed 

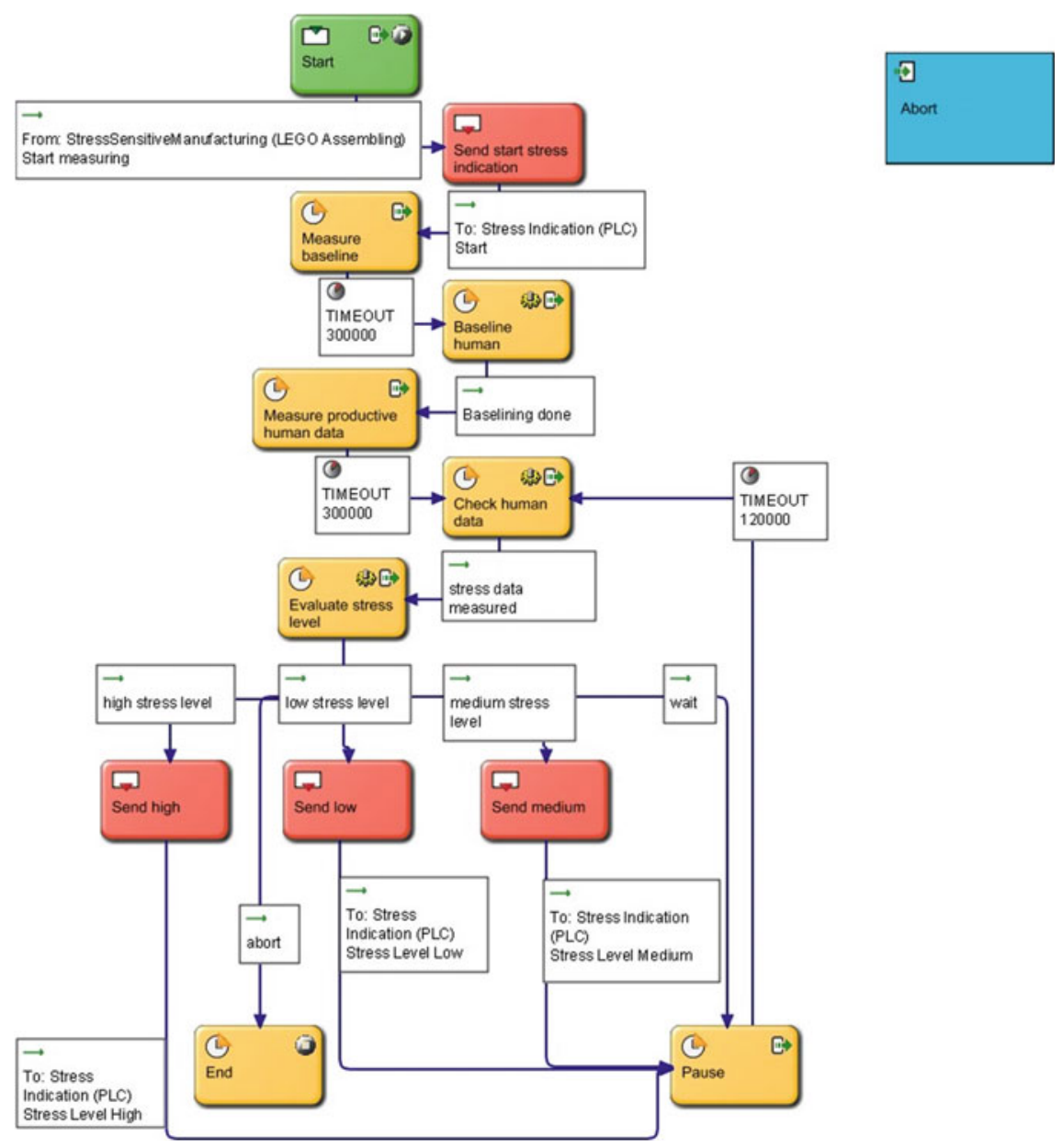

Fig. 6.12 SBD-Stress Measurement subject

by MA Systems. Hence, initial tests investigated the physiological data measured by the BioRing. In order to be able to compare measurements of the BioRing prototype, the users were asked to wear additionally a commercially available Polar H7 chest belt for measuring the heart rate (cf. http://www.polar.com/uk-en/ products/accessories/H7_heart_rate_sensor). The Polar H7 measurements were recorded via a customized Android app, in order to support ex-post data analysis and comparison.

Initially, the measurement frequency of the BioRing and the Polar H7 sensor differed. In contrast to the continuous measurement of the Polar H7 sensor, the 


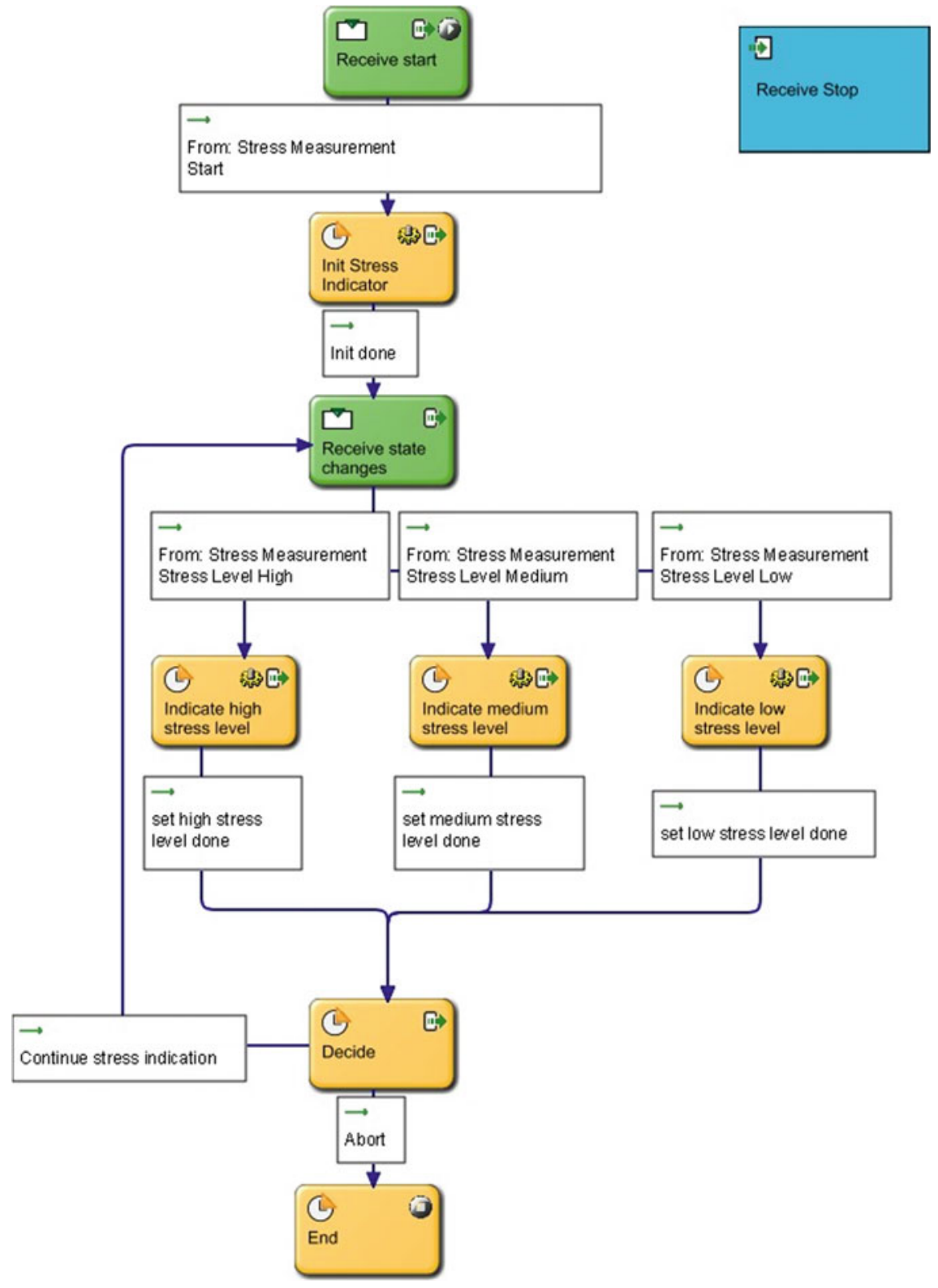

Fig. 6.13 SBD - Stress Indication subject 


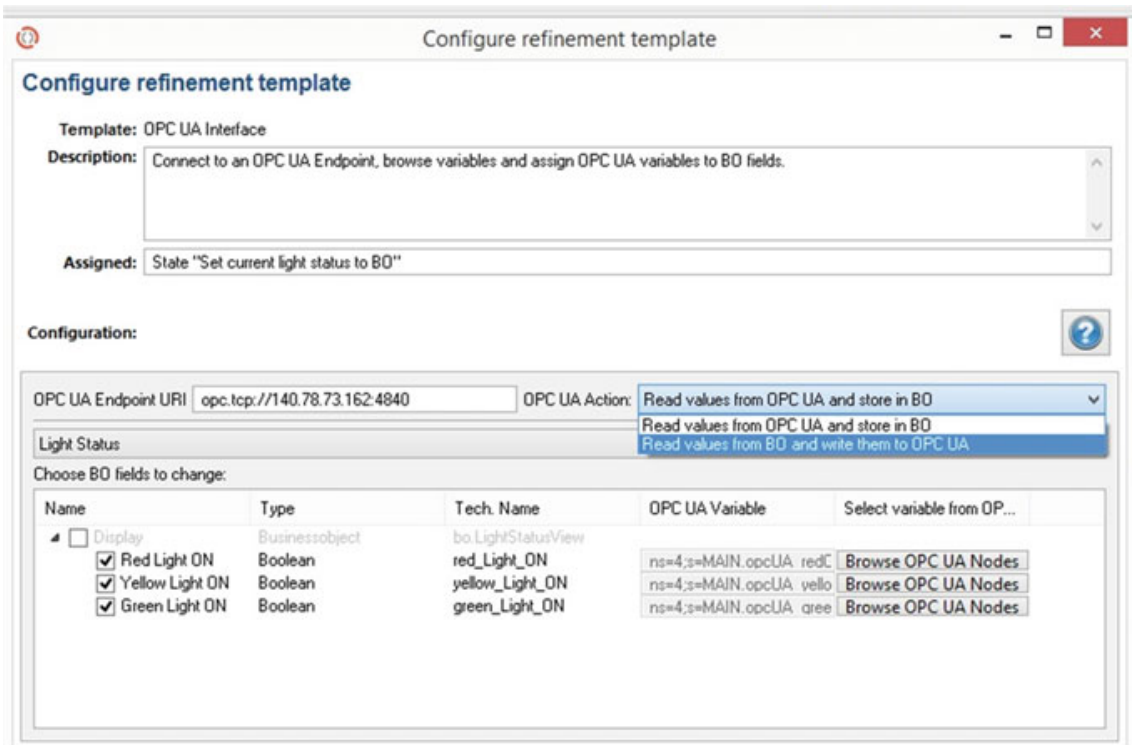

(?)

Fig. 6.14 OPC UA template for interfacing OPC UA server variables

tested BioRing prototype measured every $10 \mathrm{~s}$ for a duration of $4 \mathrm{~s}$. In order to enable the comparison of the measurements of the two different sensors, the following approach has been taken:

- Definition of measurement periods-1 period lasts $15 \mathrm{~s}$

- Calculation of the average heart rate per measurement period

Within an initial Lego assembly test three male users, aged between 20 and 35, were tested with the BioRing and the Polar $\mathrm{H} 7$ chest belt in parallel.

The initial data analysis of the BioRing compared to the commercially available Polar H7 chest belt revealed that there is still room for improvement in order to be able to apply the ring in productive settings. The degradation of the BioRing measurements during the Lego assembly phase is assumed to be due to motion artefacts, since the BioRing builds upon an optical blood flow inspection approach. These artefacts may be neglected by considering the accelerometer data provided by the ring. In the tested system, this already has been implemented. However, it decreased the number of measurements and the availability of current heart rate data.

The Lego assembly scenario itself exhibits motion, in order to accomplish the given task. Therefore, another consequence of this initial test could be to change the point of time where the stress level of a user is evaluated. In case there is a short pause in between two different tasks, the heart rate could be evaluated and compared to previous measurements during pausing situations. In general, the evaluation 


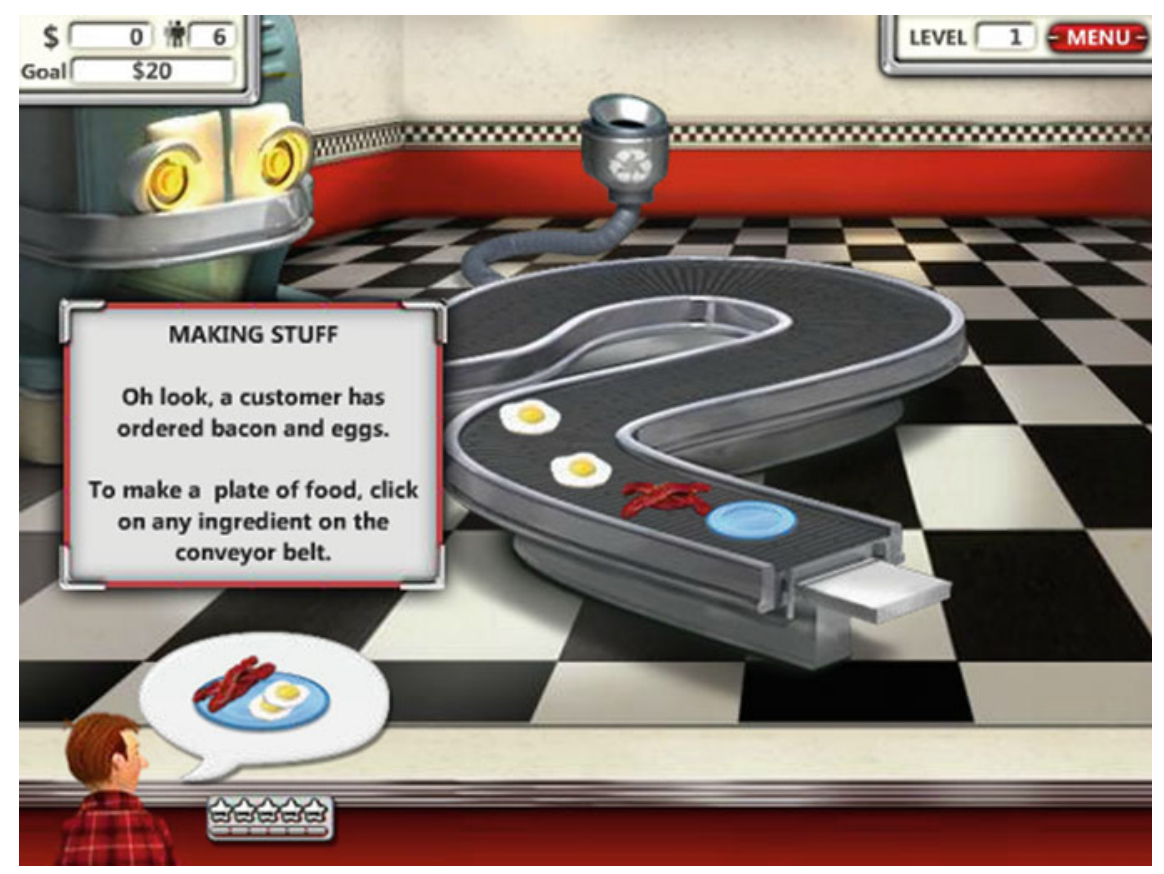

Fig. 6.15 Burger shop game to simulate occupational stress

revealed the importance of the appropriate point in time for measuring the stress level of a user with the BioRing. Another topic of improvement considered at that time concerned the measuring frequency of the BioRing. In other words, instead of measuring $4 \mathrm{~s}$ every $10 \mathrm{~s}$, data of humans should be measured continuously.

The initial findings triggered further investigations related to the applicability of the BioRing. On the one hand, the firmware of the ring was modified to continuously provide measurements, on the other hand an additional algorithm calculating the heart rate based on the measurements has been implemented. Aside from technical improvements, the application scenario has been modified, in order to avoid movements of the left hand and the left forefinger on which the BioRing typically resides. The new testing scenario applied a BurgerShop game (see Fig. 6.15) in which a player has to produce different types of orders for customers in a certain amount of time to move to the next level. Overall, the deadline to reach the top level in $20 \mathrm{~min}$ has been defined to challenge test persons.

Within this scenario, an approach similar to the previously one has been taken:

- Introduction (10 min)

- In the introduction, the test subjects get an overview of the tasks they have to accomplish. This overview includes a short explanation of the sensor setup and the game to be played.

- Equipping the test subject (5 min) 
- Test subjects wear the Polar H7 chest belt and the BioRing on the forefinger of their left hand. In order to protect the privacy, the individual subject will be asked to fix the Polar Belt $\mathrm{H} 7$ on his own at a separate room. The observers guide the participant equipping with the BioRing.

- Time of adjustment (5 min)

- Baseline data of individual subjects are collected in calm state for $5 \mathrm{~min}$.

- Playing the burger shop game (20 min)

- The subjects play the game exactly for $20 \mathrm{~min}$. The remaining time is shown in front of the PC on a large info wall. The goal is to reach level 10 within the $20 \mathrm{~min}$. In order to avoid noise w.r.t. measurement, the subjects are encouraged to keep their left hand calm and control the game via their right hand and the mouse.

Altogether 12 subjects were tested, whereby the measurements of two of them were considered invalid a priori. The reason for that was the fact that the BioRing seems to have severe issues when measuring a human with cold hands. The data resulting from such a measurement exhibit a constant heart rate of zero beats per minute. Furthermore, the width of a finger has an impact on the quality of measurements. It has been observed that skinny fingers influence negatively the quality of the measured blood flow signal. In the tests, for one subject the measurement with the BioRing did not work at all. The measurement failure was irreproducible for the testing team (neither cold or skinny finger, nor technical issues in terms of battery or service availability). Thus, only the measurements for nine subjects provided a usable data for the analysis. Subsequently, the results are described in more detail.

In the case of subjects 157,160 and 158, the Polar $\mathrm{H} 7$ only provides measured data for $15 \mathrm{~min}$ and in case of subject 160 only for $10 \mathrm{~min}$ within the human physiological database. However, this phenomenon contradicts the fact that the app continued to measure and the Polar H7 HR was shown on the display. This may be caused by network/server issues or even by service disruptions.

Overall, there were nine measurements that provided suitable data for further processing. The evaluation of these data was conducted by employing two distinct ways of calculating the HR, i.e. the old and the improved and novel algorithm. For five subjects, the new way of calculating the HR worked out properly. Properly means that the BioRing HR approximately conforms to the HR retrieved by the Polar H7. For the remaining four data sets only the original HR could be calculated. The reason for this discrepancy could not be determined.

The measured values regarding the polar belt show that the HR of the nine subjects changed during the experiment. The recorded HR varies around \pm 5.52 b.p. $\mathrm{m}$ on average. In fact, the data of all subjects exhibit a constant up and down of the HR. However, subject 154 displayed a significant increase of 28 b.p.m. above baseline compared to the average increase of 5.52 b.p.m. It has also been observed that at the end of the experiment the HR of subject 156 and 157 increased slightly by 5 b.p.m. above the average increase of 5 b.p.m, whereby, the HR of subject 158 and 159 increased significantly by $15-25$ b.p.m. The reason for this rise may be the increased stress the subjects experienced at the end of the 20 min interval. 
In summary, the experiences gained with respect to sensing stress based on heart rate show that system designers and implementers need to carefully take into account the measured data provided by human sensors. Dedicated software needs to analyze and correct wrong measurements before considering any behaviour change of an automated system based on heart rate. Finally, we can recommend involving users in system adaption based on their physiological states. This increases the awareness of users and avoids unexpected behavioural changes of the systems.

\subsubsection{Findings with Respect to Adaptive S-BPM Processes}

In the laboratory case, the sensor integration has been implemented by custom refinements for evaluating stress levels. Thus, customized Java programme code was written to evaluate the sensor data stored in a database. This approach requires developers to investigate algorithms for stress measurement based on heart rate and encode them within the models. With respect to reusability, modularity and flexibility, a middleware for human physiological data management would be beneficial. This middleware could be implemented as message bus (cf. Bernstein et al. 2009) that notifies interested processes when, e.g. changes in stress levels or location occur. Given such a middleware, it could be applied in dedicated subject behaviours of adaptive S-BPM processes. Guidelines for designing such processing systems are derived subsequently.

The related work on adaptive systems described above and multiple process developer discussions within the SO-PC-Pro project inspired the formulation of design guidelines for adaptive S-BPM processes. These findings are summarized in the following, in order to provide a reference point for process analysts, designers and implementers. The related work (cf. Sect. 6.1) revealed two important aspects when designing adaptive systems:

- Means for context perception and assessment, and

- Means for adapting different workplace elements adequately

The former refers to the presented triggers of adaptation, whereas the latter refers to the taxonomy of adaptations. In the LEGO assembly scenario, the measurement of physiological attributes of an operator has been investigated as enabler triggering system adaptation based on different stress levels. The simple test model uses the subject "Stress measurement" to continuously perform both checking and evaluating heart rate data for a person based on data stored within a central database.

Thus, the "Stress measurement" subject needs to pro-actively check and evaluate. Even if this approach worked within the laboratory setting, a re-active approach might be an alternative in productive settings (reduction of traffic, running processes, number of requests to the DB...). Re-active means that the S-BPM process is notified from an external "human-sensing" middleware in case of changes (e.g. stress low, stress high...) and does not continuously check for changes itself. This middleware could also manage the sensors used to measure human data (e.g. single measurement by one sensor, multiple sensors providing same values). 
Aside from monitoring operators via sensors to trigger adaptation, operators may also pro-actively trigger adaptations, e.g. by dedicated functionalities like request job rotation, pause or task offloading. For this reason, the triggering behaviour needs to be modelled within the internal behaviour of the subjects.

By enabling the integration of PLC behaviour and sensors in S-BPM processes also environmental states at a smart workplace like lighting, temperature or table height may be observed and applied to trigger process changes.

Spatio-temporal aspects (time, location) may also be considered in process designs. Time may either be considered in timer-events, measurement of times takes for certain operations. Location may be provided manually via user input, automatically via GPS or scanning of worker RFID tag.

System triggers and task/mission triggers may be directly encoded in S-BPM models (in transitions, rules). Thus, monitoring of the process progress, e.g. via KPIs or current process state, allows to check an S-BPM processing system state.

Adaptation may affect function allocation, task scheduling, interaction and content. In S-BPM, functions are performed by certain subjects or processes. A modeller may define dedicated roles and users at design time and thus allocate humans or machines in advance to performing the behaviour at runtime. This would correspond to static function allocation. In case a process designer wants to implement a dynamic allocation of humans and machines to subjects at runtime two means may help: (i) selection of an actor out of a list of actors assigned to the role and (ii) dynamic process binding in case different behaviours (e.g. machine behaviour vs. human behaviour) may be triggered when a certain task needs to be accomplished.

Scheduling of tasks in S-BPM may be realized via a dedicated Scheduling subject (either human or machine, or both) which instantiates processes, prioritizes them, defines aimed target duration, monitors tasks completion and modifies schedule based on operation outcomes. In an S-BPM process Timing relates to the point of time where a task/process is initiated. This timing may be affected by manual process instantiation, instantiation upon message receipt or system triggered instantiation.

Duration refers to the time a task takes. In S-BPM, task durations of individual subjects may be monitored and KPIs defined to support the management via the indication of deviations. Furthermore, S-BPM-based simulation models could be used gain insight in potential system behaviour before adapting/rescheduling operations. Aside duration, Priorization of tasks may be encoded in S-BPM processes in dedicated Business Object fields that are evaluated at runtime.

Another element affected by modifications within adaptive systems represents the interaction among users and machines. S-BPM processes may be tailored to different user groups. Furthermore, recent developments (Kannengiesser et al. 2016) allow customizing user interfaces according to different types of users. In close relation with the interaction, developers need to consider the content to be displayed to users. In S-BPM a designer would need to consider different codalities, 
levels of abstraction and the quality within the related business objects. This would allow for device- and user-specific shipping of content.

With respect to the taxonomy of adaptive systems, the following generic types of subjects may be derived (cf. Fig. 6.1).

\section{Generic context assessment subjects}

- Task identifier

- Who (man or machine) is doing what?

- Human property sensing

- What is the current value of relevant human properties (e.g. stress level, state of exhaustion...)?

- Human state inference

- What is the current state of a human (e.g. working, pausing, running...)?

- Machine property sensing

- What is the current value of relevant machine properties (e.g. power consumption, acceleration, speed...)?

- Machine state inference

- What is the current state of a machine (e.g. idle, standby, producing...)?

- Environmental property sensing

- What is the current value of relevant environmental properties (e.g. humidity, temperature, illumination...)?

- Environmental state inference

- What is the current environmental state (e.g. hot and humid, daylight, artificial lighting...)?

\section{Generic adaptation subjects}

- Task allocation - refers to the above given concept of "Function allocation" and considers who (human or machine) is responsible to perform a certain function or task. This may be encoded in static rules within the process models or dynamically decided during runtime based on the current work distribution among humans and machines 
- Task scheduling - such a subject defines the interface among a scheduling software focusing on (timing, duration, prioritization) and the processes to be triggered or dynamically reallocated

Dedicated subjects for adapting interaction and content are not recommended. Regarding the adaptation of interaction, the defined interaction logic resides in S-BPM within the defined subject interactions and the exchanged messages. Thus, dedicated models need to be created with respect to different interactions and provided for execution support. Regarding content adaptation, one could model a dedicated "content broker" that provides for different types of consuming subjects adequate content, e.g. in terms of quantity, or granularity. Alternatively, dedicated content-provision subjects could be defined for each type of content consumer.

Based on the individual project, the given subjects and intended behaviour may be considered by process designers to implement adaptive S-BPM processing systems. The assessment subjects are split-up into subjects sensing concrete values and subjects responsible for inferring a certain state. Thereby, multiple sensors could provide data on relevant properties and the inference subjects may consider them. This separation also allows interested subject to independently request current values of certain properties and inferred states. Furthermore, subjects aggregating the different states of humans, machines and the environment may be modelled in future process development projects since these dimensions typically need to be carefully aligned.

\subsection{Conclusive Summary}

The worker and his or her well-being is regarded a crucial part of manufacturing situations. Designing and implementing adaptive systems considering human properties to identify disturbances, monitor crucial states and tune workplaces a.t.l., in order to fit human needs gained interest in diverse research areas, such as human factors engineering, pervasive computing, Industry 4.0 or factories of the future.

Advances in the area of wearable sensors proclaim that sensing human properties within manufacturing settings is technically feasible. Thereby, sensing human properties, such as the level of comfort or stress, may be used to adapt system behaviour in manufacturing situations. In this chapter, S-BPM's capabilities to design a human-aware assembly scenario have been investigated. Thereby, related work from adaptive systems design addresses dimensions and triggers for adaptation. We could derive relevant design aspects and set up a laboratory environment at the Johannes Kepler University Linz for testing measurements and adaptation designs. The developed system architecture and the respective S-BPM models can be used for further projects due to the generic integration approach of S-BPM with sensors via Web Services and the OPC Unified Architecture (cf. Neubauer et al. 2015). 
Although measuring human physiological data in work situations is technically feasible, designing such systems requires ensuring the availability and reliability of human sensor data. The laboratory test revealed that even commercially available sensors like the Polar $\mathrm{H} 7$ chest belt may fail in terms of delivering no measurements due to lack of contact with the skin. Hence, for certain work situations it is recommended to investigate suitable sensors and their applicability within the given work environment. Furthermore, we could observe that the quality of the BioRing measurements differs among test subjects. Influencing factors like finger temperature or width of fingers cause different quality of heart rate measurements. Therefore, a "one-sensor fits them all" mentality is not appropriate. We rather recommend evaluating the appropriateness of certain sensors with individual humans in concrete work settings.

Adaptive processing systems can be implemented utilizing S-BPM concepts and technologies. Adaptation may either be triggered explicitly by humans or implicitly via sensors. Certain machine states, environmental or spatio-temporal states may trigger adaptation. Triggers may cause changes in task allocation and task scheduling. Finally, the interaction between users and the system, as well as the content provided may be adapted. Possibilities to considerer the triggers and types of adaptations in S-BPM models have been discussed in this chapter. They shall serve as starting point for future developments targeting adaptive sensor-based $\mathrm{S}-\mathrm{BPM}$ processes initiating changes at runtime.

\section{References}

Bernstein, D., Ludvigson, E., Sankar, K., Diamond, S., \& Morrow, M. (2009). Blueprint for the intercloud-protocols and formats for cloud computing interoperability. In M. Perry, H. Sasaki, M. Ehmann, G. O. Bellot, \& O. Dini (Eds.), The Fourth International Conference on Internet and Web Applications and Services ICIW 2009 (pp. 328-336). IEEE. doi:10.1109/ICIW.2009.55.

Boblin, G. (1976). Delayed habituation of the electrodermal orienting response as a function of increased level of arousal. Psychophysiology, 13, 345-351. doi:10.1111/j.1469-8986.1976. tb03088.x.

Feigh, K. M., Dorneich, C., \& Hayes, C. C. (2012). Toward a characterization of adaptive systems: A framework for researchers and system designers. Human Factors, 54(6-12), 1008-1024. doi:10.1177/0018720812443983.

Jacobs, S. C., Friedman, R., Parker, J. D., Tofler, G. H., Jimenez, A. H., Muller, J. E., et al. (1994). Use of skin conductance changes during mental stress testing as an index of autonomic arousal in cardiovascular research. American Heart Journal, 128(6), 1170-1177.

Jouven, X., Schwartz, P. J., Escolano, S., Straczek, C., Tafflet, M., Desnos, M., et al. (2009). Excessive heart rate increase during mild mental stress in preparation for exercise predicts sudden death in the general population. European Heart Journal, 30, 1703-1710.

Kannengiesser, U., Heininger, R., Gründer, T., \& Schedl, S. (2016). Modelling the process of process execution: A process model-driven approach to customising user interfaces for business process support systems. In International Workshop on Business Process Modeling, Development and Support (pp. 34-48). Springer International Publishing. doi:10.1007/978-3319-39429-9_3. 
Kelsey, R. M. (1991). Electrodermal lability and myocardial reactivity to stress. Psychophysiology, 28(6), 619-631. doi:10.1111/j.1469-8986.1991.tb01005.x.

Lawler, K. A. (1980). Cardiovascular and electrodermal response patterns in heart rate reactive individuals during psychological stress. Psychophysiology, 17(5), 464-470. doi:10.1111/j. 1469-8986.1980.tb00185.x.

Lundberga, U., Granqvistb, M., Hanssonc, T., Magnussonc, M., \& Wallind L. (1989). Psychological and physiological stress responses during repetitive work at an assembly line. Work \& Stress: An International Journal of Work, Health \& Organisations, 3(2).

NASA Langley Technical Report. Retrieved July 20, 2016, from http://ntrs.nasa.gov/archive/nasa/ casi.ntrs.nasa.gov/20010067614.pdf.

Neubauer, M., Krenn, F., \& Majoe, D. (2015). Towards an architecture for human-aware modeling and execution of production processes. IFAC-PapersOnLine, 48(3), 294-299.

O'Gorman, J. G., \& Homeman, C. (1979). Consistency of individual differences in nonspecific electrodermal activity. Biological Psychology, 9(1), 13-21. doi:10.1016/0301-0511(79)90019-X.

Scerbo, M. W., Parasuraman, R., Di Nocero, F., \& Prinzel, L. J. (2001). The efficacy of psychophysiological measures for implementing adaptive technology. NASA/TP-2001211018.

Steinhauser, N. B., Pavlas, D., \& Hancock, P. A. (2009). Design principles for adaptive automation and aiding. Ergonomics in Design, 17(2), 6-10.

Taelman, J., Vandeput, S., Spaepen, A., \& Van Huffel, S. (2009). Influence of mental stress on heart rate and heart rate variability. In J. Vander Sloten, P. Verdonck, M. Nyssen, \& J. Haueisen (Eds.), 4th European Conference of the International Federation for Medical and Biological Engineering, IFMBE Proceedings (Vol. 22, pp. 1366-1369). Berlin: Springer.

Van der Klink, J. J., Blonk, R. W., Schene, A. H., \& Van Dijk, F. J. (2001). The benefits of interventions for work-related stress. American Journal of Public Health, 91(2), 270-276.

Open Access This chapter is distributed under the terms of the Creative Commons AttributionNonCommercial 4.0 International License (http://creativecommons.org/licenses/by-nc/4.0/), which permits any noncommercial use, duplication, adaptation, distribution and reproduction in any medium or format, as long as you give appropriate credit to the original author(s) and the source, provide a link to the Creative Commons license and indicate if changes were made.

The images or other third party material in this chapter are included in the work's Creative Commons license, unless indicated otherwise in the credit line; if such material is not included in the work's Creative Commons license and the respective action is not permitted by statutory regulation, users will need to obtain permission from the license holder to duplicate, adapt or reproduce the material. 